\title{
Intracellular Modelling of Cell-Matrix Adhesion during Cancer Cell Invasion
}

\author{
V. Andasari and M.A.J. Chaplain * \\ Division of Mathematics, University of Dundee, DD1 4HN Dundee, Scotland UK
}

\begin{abstract}
When invading the tissue, malignant tumour cells (i.e. cancer cells) need to detach from neighbouring cells, degrade the basement membrane, and migrate through the extracellular matrix. These processes require loss of cell-cell adhesion and enhancement of cell-matrix adhesion. In this paper we present a mathematical model of an intracellular pathway for the interactions between a cancer cell and the extracellular matrix. Cancer cells use similar mechanisms as with normal cells for their interactions with the extracellular matrix. We develop a model of cell-matrix adhesion that accounts for reactions between the cell surface receptor integrins, the matrix glycoprotein fibronectin, and the actin filaments in the cytoskeleton. Each represents components for an intermediate compartment, the extracellular compartment, and the intracellular compartment, respectively. Binding of fibronectin with integrins triggers a clustering of protein complexes, which then activates and phosphorylates regulatory proteins that are involved in actin reorganisation causing actin polymerization and stress fibre assembly. Rearrangement of actin filaments with integrin/fibronectin complexes near adhesion sites and interaction with fibrillar fibronectin produces the force necessary for cell migration, accounting for cell-matrix adhesion.
\end{abstract}

Key words: cancer invasion, cell-matrix adhesion, integrins, fibronectin, actin AMS subject classification: 92C10, 92C17, 92C 37, 92C40, 92C50

\section{Introduction}

Adhesive interactions between cancer cells and their surroundings, i.e., the extracellular matrix, are vital for metastatic spread. The adhesive interactions are required for migration through the

*Corresponding author. E-mail: vivi@maths.dundee.ac.uk 
basement membrane and extracellular matrix surrounding the tumour after detachment from the primary tumour mass, and for penetration of the circulating tumour cells into the tissue of the target organ at the secondary site.

In the interactions between cell and matrix, termed cell-matrix adhesion, the intracellular compartment of the cell is connected with the extracellular environment through cell surface receptors. Inside the cell, cell surface receptors are indirectly linked to the actin cytoskeletal network that is dynamically remodelled. Outside the cell, cell surface receptors bind with extracellular matrix components such as fibronectin. The reorganisation of the actin cytoskeleton combined with binding of cell surface receptors to matrix components provides a mechanical linkage between cell and matrix, to produce the force or traction necessary for cell adhesion, spreading and migration.

Cancer cells migrate in various ways, according to cell type and degree of differentiation. The molecular mechanisms underlying the migration of cancer cells through the tissues are similar to the mechanisms used by normal, non-neoplastic cells for migration during physiological processes, such as wound healing, embryonic morphogenesis, immune cell trafficking, etc. The principles of cell migration were initially investigated in non-neoplastic fibroblasts, keratinocytes and myoblasts, but additional studies on tumour cells show that the same basic strategies are retained [13].

Cell migration is an integrated process that requires the continuous, coordinated formation and disassembly of adhesions. Migration can be viewed as a cyclical process. Based on studies, it is commonly agreed that the basic migratory cycle includes the following processes $[36,26,32,49$, 4]:

- Extension of a protrusion. Polymerisation of the actin cytoskeletal network drives the initial extension of the plasma membrane at the cell front;

- Formation of stable contacts near the leading edge of the protrusion. The interaction of the cell surface receptors with the extracellular matrix stabilises the adhesions by recruiting signalling and cytoskeletal proteins;

- Cytoskeletal contraction, that generates a tractional force;

- Translocation of the cell body forward. The small, nascent adhesions may transmit strong forces, and serve as traction points for the propulsive forces that move the cell body forward; and

- Release of adhesions and retraction at the cell rear. This completes the migratory cycle allowing net translocation of the cell in the direction of movement.

The major groups of cell surface receptors for mediating cell-matrix adhesion are known as integrins, named for their role in integrating the intracellular cytoskeleton with the extracellular matrix [33]. Integrins provide a bi-directional signalling for mechanochemical information across the cell membrane, that is, two-way signalling occurs from outside-to-inside and from inside-tooutside. This signalling functioning provides a major mechanism for connecting the intracellular compartment (which is the cytoskeleton) and the extracellular compartment (extracellular matrix components or glycoproteins, such as fibronectin). 
Over the last twenty years there have been attempts to mathematically model the interaction between cells and surfaces representing the extracellular matrix, such as by [21] who modelled cell adhesion to surfaces mediated by specific binding between molecules on the cell surface and complementary ligand molecules on the receiving surface. The binding depends on various quantities such as receptor number, binding affinity between receptor and ligand, bond formation rate, receptor diffusivity, distractive fluid forces, and contact area. [35] modelled the interactions between integrins, the cytoskeleton, and the matrix for cell retraction and dissociation mechanisms at the cell rear. [11] modelled the dependence of cell speed on adhesion-receptor/ligand binding and cell mechanical properties. Their model incorporated cytoskeletal force generation, cell polarization, and dynamic adhesion for cell movement.

In this paper we model a cell-matrix adhesion pathway describing interactions between fibronectin, integrins and actin reorganisation. Binding of fibronectin with integrins triggers a clustering of protein complexes, which then activates and phosphorylates regulatory proteins that are involved in actin reorganisation causing actin polymerisation and stress fibre assembly. Rearrangement of actin filaments with integrin/fibronectin complexes near adhesion sites and interaction with fibrillar fibronectin produces the force necessary for cell migration, accounting for cell-matrix adhesion.

\section{Structural Components of Cell-matrix Adhesion}

To model the mechanisms of cell adhesion to extracellular matrix, we consider three major components that are needed to generate traction used by cells to perform migration through the matrix. These components are the cell surface receptors integrins, the matrix glycoprotein fibronectin, and the actin cytoskeleton.

Integrins comprise a large family of transmembrane glycoproteins that mainly function to mediate cell-matrix adhesion. Integrins are composed of two subunits, $\alpha$ and $\beta$, and each $\alpha \beta$ combination has its own binding specificity and signalling properties [18]. To date, there are $18 \alpha$ and $8 \beta$ subunits which combine with each other to form a family of 24 distinct heterodimeric integrins that have been identified in humans $[22,46]$. Heterodimeric integrins are expressed on the cell membrane to link the actin cytoskeleton to the extracellular matrix. Three different $\beta_{1}$ integrins, which are $\alpha_{4} \beta_{1}, \alpha_{5} \beta_{1}$, and $\alpha_{8} \beta_{1}$, have been found which bind to fibronectin. Another large subfamily of integrins is composed of heterodimers sharing the $\alpha_{V}$ subunit, also receptors for fibronectin, $\alpha_{V} \beta_{1}$, $\alpha_{V} \beta_{3}, \alpha_{V} \beta_{5}, \alpha_{V} \beta_{6}$, and $\alpha_{V} \beta_{8}$ [24].

The cytoplasmic tails of integrins are generally short with roughly 50 amino acids in length, except for integrin $\beta_{4}$ that has $\sim 1000$ amino acids. The cytoplasmic tails are also devoid of enzymatic features. Hence, integrin cytoplasmic domains form multi-molecular complexes with proteins involved in cell signalling and adapter proteins that connect the integrin to the actin cytoskeletal system, cytoplasmic kinases, and transmembrane growth factor receptors $[18,5]$. The cytoskeleton organisation is performed after accumulation of cytoskeletal proteins like tensin, $\alpha$ actinin, talin, vinculin, and focal adhesion kinase (FAK). 
Migrating cells bind to fibronectin by means of discrete integrin clusterings at the leading or protruding edge. Integrin clustering can be a result of integrin binding with fibronectin which then promotes the localisation of adaptor proteins linking to the cytoskeleton $[18,5]$ or the clustering of integrins can cause binding of the leading edge of the cell with fibronectin. A study on integrin clusterings in motile fibroblasts by [41] showed that integrin clusterings grow at the leading edge and shrink at the cell rear over time, in the sense that they fluctuate in size, density, and shape over a period of minutes. As cells move, integrin clusterings that bind with fibronectin (often referred to as focal contacts or focal adhesion sites) appear at the leading edge and diminish in number toward the cell centre, while at the cell rear there is only a small number of clusterings that abruptly break as the cell body advances forward. Experiments carried out by [9] demonstrated that integrin activation is a prerequisite for clustering events. They observed that integrin clusterings in the periphery of the cell occur within minutes, starts about after 3 minutes. There are also many recent studies on integrin clustering, such as by [19, 48, 39, 52, 55, 2, 25, 45].

Integrin binding with matrix can also affect integrin activation, where integrin binding to fibronectin changes the conformational state of integrin, from inactive to active $[17,16,5]$. Integrin activation is a prerequisite for clustering, hence activated integrins preferentially localise to the leading edge, where new adhesions form. In our model, we assume that integrin activation is triggered by binding of integrin to fibronectin, which then leads to clustering of integrin/fibronectin complexes.

The clustering of integrin/fibronectin complexes at the focal adhesion sites regulates downstream signalling pathways that induce an accumulation of signalling and adaptor proteins near the cytoplasmic domains of integrins. There are more than 50 cytoplasmic proteins present in the cellmatrix adhesion structure. The downstream signals coordinate reorganisation of the cytoskeleton as a result of actin polymerisation. The cytoskeleton is a highly dynamic part of a cell. It consists of numerous cytoskeletal actin filaments (F-actin) that constantly grow at their barbed end through polymerisation of monomeric actin (G-actin) and shrink at the pointed end through actin depolymerisation. This process is referred to as "treadmilling", which is a steady state flux of filaments $[40,51]$. The reorganisation of the actin cytoskeleton is the primary mechanism of cell motility and is essential for most types of cell migration. During cell migration, the actin cytoskeleton is dynamically remodelled, and this reorganisation produces the force necessary for cell migration. The reorganisation of actin filaments into larger stress fibres, in turn, causes more integrin clusterings, thus enhancing the matrix binding and organisation by integrins in a positive feedback system [18]. Evidence accumulated from a variety of cell types has shown that the actin filaments assembled near the leading edge are also transported rearward or in a direction opposite to the movement of the cell. This phenomenon is known as retrograde flow. There is a balance between the actin cytoskeleton assembly and the retrograde flow that govern and control cell movement $[1,27,54,20]$.

The structure and composition of the extracellular matrix is also very important for cancer cell invasion. One of the key components of the extracellular matrix which plays a major role in cell-matrix adhesion is fibronectin. Two forms of fibronectin exist. One form, plasma or soluble fibronectin, is a major plasma protein and found at concentration of $300 \mu \mathrm{g} / \mathrm{ml}$ in human blood. An early study by [47] showed that hepatocytes of rats and hamster synthesised and secreted fi- 
bronectin as a soluble dimeric protein. In humans, soluble fibronectin is synthesised predominantly in the liver by hepatocytes in dimeric form. Another form, known as cellular fibronectin, is found at the surfaces of many different cell types, where it forms insoluble fibrillar matrices [38]. A major fraction of the cellular fibronectin in the extracellular matrix has been found to be derived from plasma fibronectin [30]. The assembly of fibronectin matrix or insoluble fibrillar matrix is a cell-mediated process. It is thought that the binding of integrin to fibronectin promotes fibrillar matrix formation through interactions with other cell-association fibronectin dimers. As fibrillar matrices form on the outside of the cell, cytoplasmic domains of integrins organise cytoplasmic proteins (signalling and adaptor proteins) into functional complexes linking to the cytoskeleton. The whole process of binding of cytoskeleton to integrins and fibronectin is essential for fibrillar matrices formation and propagation [53]. The insoluble fibrillar matrices, along with other components of extracellular matrix, provide structural support for cellular functions such as cell adhesion, migration, and tissue organisation. For migrating cells, binding onto a rigid surface helps generate tractional forces necessary for moving the cell body forward. Since the assembly of fibronectin into a fibrillar matrix is a complex process, we assume, for our model, that the fibrillar matrices are formed by binding of the clustering of integrin/fibronectin complex bound with soluble fibronectin. The more fibronectin or fibrillar matrices formed, the more rigid the extracellular matrix becomes. The rigidity of extracellular matrix causes the strengthening of the integrin-cytoskeleton linkages [8]. Hence it generates positive feedback for cytoskeleton organisation inside of the cell. The adhesion forces are the final step produced from the kinetics and mechanics of the structural components of cell-matrix adhesion.

\section{Mathematical Model Derivation}

Based on the kinetics of the major components of cell-matrix adhesion summarised in section 2., we assume the following steps for our cell-matrix adhesion pathway:

1. Binding of integrin $[R]$ to plasma or soluble fibronectin $\left[F_{p}\right]$ to form an integrin/fibronectin complex [FR], with reversible reactions:

$$
\mathrm{R}+\mathrm{F}_{\mathrm{p}} \rightleftharpoons{ }_{k_{2}}^{k_{1}} \mathrm{FR}
$$

2. The clustering of integrin/fibronectin complex results from the binding of integrin with fibronectin at the cell membrane. Here we adopt a simple clustering reaction as in [14], which is assumed to be irreversible, with rate $k_{3}$ :

$$
\mathrm{FR} \rightarrow{ }^{k_{3}} \widetilde{\mathrm{FR}}
$$

3. Clustering triggers recruitment and localisation of cytoplasmic proteins, hence activation and phosphorylation of regulating proteins. This then activates actin polymerisation and actin reorganisation $A_{p}$ at the leading edge that pushes the membrane forward in finger-like 
structures (filopodia) and can also be in sheet-like structures (lamellipodia), with rate $k_{41}$. To account for processes that reduce actin filaments at the leading edge such as treadmilling and retrograde flow, we assume reverse reaction (depolymerisation) with rate $k_{42}$ and natural decay with constant rate $m$.

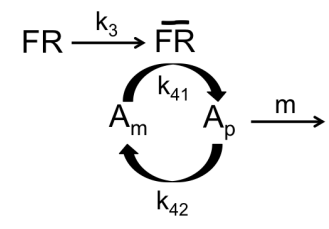

4. Rearrangement of actin filaments with clusters of the integrin/fibronectin complex near focal contact sites:

$$
\mathrm{A}_{\mathrm{p}}+\widetilde{\mathrm{FR}} \rightleftharpoons{ }_{k_{6}}^{k_{5}} \mathrm{~A}_{\mathrm{p}} \cdot \widetilde{\mathrm{FR}}
$$

5. Meanwhile, surrounding the cell in the extracellular domain, the formation of the fibrillar fibronectin network occurs with a rate $k_{7}$ :

$$
\widetilde{\mathrm{FR}}+\mathrm{F}_{\mathrm{p}} \rightarrow^{k_{7}} \mathrm{~F}_{\times}
$$

The formation of the fibrillar fibronectin network increases matrix rigidity, which in turn, gives a positive feedback with rate $k_{8}$ to the strengthening of the integrin/fibronectin-cytoskeleton linkage $A_{p} . \widetilde{F R}$.

6. Binding of the integrin/fibronectin-cytoskeleton linkage $\mathrm{A}_{\mathrm{p}} . \widetilde{\mathrm{FR}}$ to the fibrillar network yields an adhesion force, measured by its strength, required for cell movement:

$$
\mathrm{F}_{\times}+\mathrm{A}_{\mathrm{p}} \cdot \widetilde{\mathrm{FR}} \rightleftharpoons{ }_{k_{10}}^{k_{9}} \mathrm{~F}_{\times} \cdot \mathrm{A}_{\mathrm{p}} \cdot \widetilde{\mathrm{FR}}
$$

The complex $F_{\times} \cdot A_{p} \cdot \widetilde{F R}$ ruptures during cell detachment at the cell rear. Hence the reaction is reversible.

7. An increasing force adds a positive feedback to the clustering of complex with rate $k_{11}$.

\begin{tabular}{|c|c|c|}
\hline [R] & $=x_{1}$ & (free integrin) \\
\hline$\left[\mathrm{F}_{\mathrm{p}}\right]$ & $x_{2}$ & (soluble fibronectin) \\
\hline [FR] & $x_{3}$ & (integrin/fibronectin complex) \\
\hline$[\widetilde{\mathrm{FR}}]$ & $x_{4}$ & (clustering of integrin/fibronectin complex) \\
\hline$\left[\mathrm{A}_{\mathrm{p}}\right]$ & $x_{5}$ & (actin polymerisation) \\
\hline$\left[\mathrm{A}_{\mathrm{p}} \cdot \widetilde{\mathrm{FR}}\right]$ & $x_{6}$ & (integrin/fibronectin-cytoskeleton linkage) \\
\hline$\left[\mathrm{F}_{\times}\right]$ & $x_{7}$ & (insoluble or fibrillar fibronectin) \\
\hline$\left[\mathrm{F}_{\times} \cdot \mathrm{A}_{\mathrm{p}} \cdot \widehat{\mathrm{FR}}\right]$ & $=x_{8}$ & (adhesion force) \\
\hline$\left[\mathrm{A}_{\mathrm{m}}\right]$ & $=x_{9}$ & (actin monomer) \\
\hline
\end{tabular}

These intracellular interactions are summarised in a schematic diagram shown in Fig. 1.

By letting 


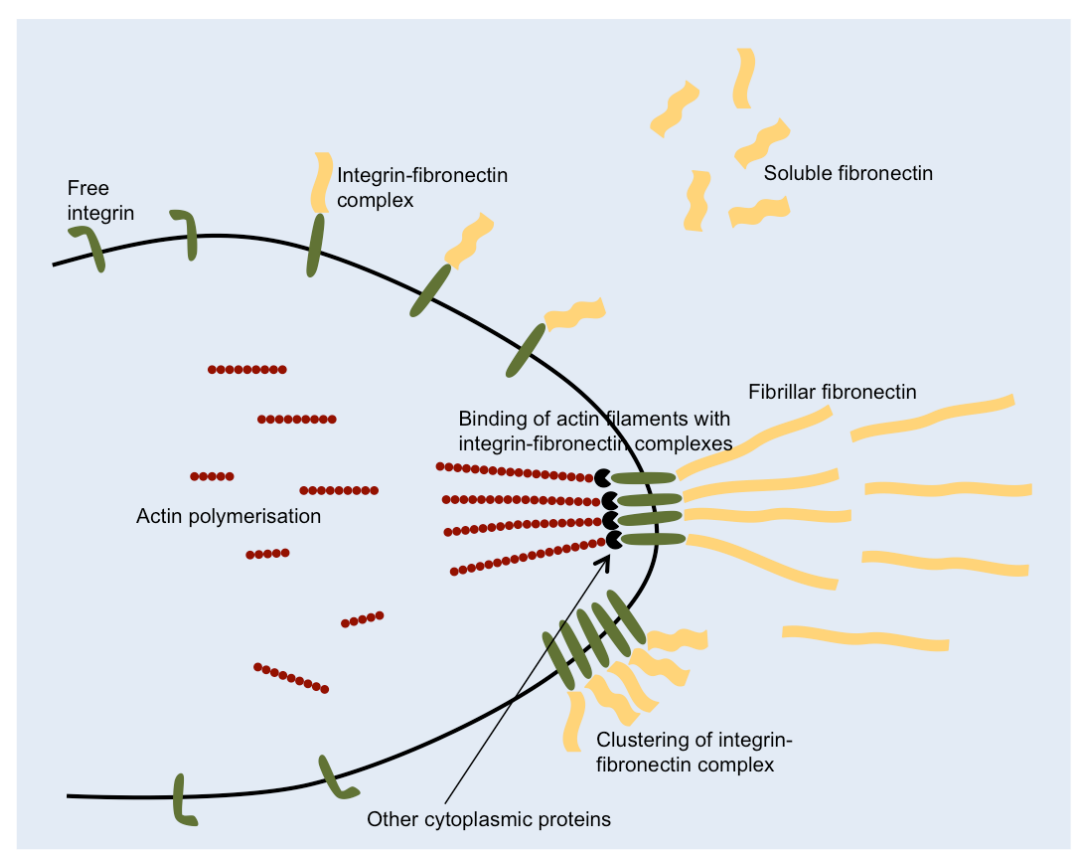

Figure 1: Schematic diagram showing the key processes of cell-matrix adhesion involving integrin, fibronectin and actin.

and applying the law of mass action, we obtain the system of ODEs:

$$
\begin{aligned}
& \frac{d x_{1}}{d t}=k_{2} x_{3}-k_{1} x_{1} x_{2}, \\
& \frac{d x_{2}}{d t}=k_{2} x_{3}-k_{1} x_{1} x_{2}-k_{7} x_{2} x_{4}, \\
& \frac{d x_{3}}{d t}=k_{1} x_{1} x_{2}-k_{2} x_{3}-k_{3} x_{3}, \\
& \frac{d x_{4}}{d t}=k_{3} x_{3}+k_{6} x_{6}-k_{5} x_{4} x_{5}-k_{7} x_{2} x_{4}+k_{11} x_{8}, \\
& \frac{d x_{5}}{d t}=k_{41} x_{9}-k_{42} x_{5}+k_{6} x_{6}-k_{5} x_{4} x_{5}-m x_{5}, \\
& \frac{d x_{6}}{d t}=k_{5} x_{4} x_{5}+k_{8} x_{7}-k_{6} x_{6}-k_{9} x_{6} x_{7}+k_{10} x_{8}, \\
& \frac{d x_{7}}{d t}=k_{7} x_{2} x_{4}-k_{8} x_{7}+k_{10} x_{8}-k_{9} x_{6} x_{7},
\end{aligned}
$$




$$
\begin{aligned}
\frac{d x_{8}}{d t} & =k_{9} x_{6} x_{7}-k_{10} x_{8}-k_{11} x_{8} \\
\frac{d x_{9}}{d t} & =k_{42} x_{5}-k_{41} x_{9} .
\end{aligned}
$$

We introduce dimensionless variables

$$
x_{1}^{*}=\frac{x_{1}}{R_{T}}, \quad x_{2}^{*}=\frac{x_{2}}{F_{T}}, \quad x_{5}^{*}=\frac{x_{5}}{R_{T}}, \quad x_{9}^{*}=\frac{x_{9}}{R_{T}},
$$

where $R_{T}$ is the total integrin concentration $\left(R_{T}=[\mathrm{R}]+[\mathrm{FR}]+[\widetilde{\mathrm{FR}}]\right)$ and $F_{T}$ is the total ligand/fibronectin concentration $\left.\left(F_{T}=\left[\mathrm{F}_{\mathrm{p}}\right]+[\mathrm{FR}]+\widetilde{\mathrm{FR}}\right]+\left[\mathrm{F}_{\times}\right]\right)$. To set the reference for concentration of integrin/fibronectin complex, we follow [16], that under conditions of excess ligand (of fibronectin) the steady state concentration of integrin/fibronectin complex is proportional to

$$
x_{F R}=K_{a} R_{T} F_{T},
$$

where $K_{a}=\frac{k_{1}}{k_{2}}$ is the equilibrium affinity constant for binding of integrins with fibronectin. We use $x_{F R}$ to define the rest of the dimensionless variables:

$$
x_{3}^{*}=\frac{x_{3}}{x_{F R}}, \quad x_{4}^{*}=\frac{x_{4}}{x_{F R}}, \quad x_{6}^{*}=\frac{x_{6}}{x_{F R}}, \quad x_{7}^{*}=\frac{x_{7}}{x_{F R}}, \quad \text { and } \quad x_{8}^{*}=\frac{x_{8}}{x_{F R}} .
$$

We nondimensionalise time as

$$
t^{*}=\frac{t}{T}
$$

where $T$ is an appropriate reference time. Using $x_{F R}$ and $T$ we obtain the nondimensional parameters:

$$
\begin{array}{llll}
k_{1}^{*}=x_{F R} k_{1} T, & k_{2}^{*}=k_{2} T, & k_{3}^{*}=k_{3} T, & k_{41}^{*}=k_{41} T, \quad k_{42}^{*}=k_{42} T, \\
k_{5}^{*}=x_{F R} k_{5} T, & k_{6}^{*}=k_{6} T, & k_{7}^{*}=x_{F R} k_{7} T, & k_{8}^{*}=k_{8} T, \\
k_{9}^{*}=x_{F R} k_{9} T, & k_{10}^{*}=k_{10} T, & k_{11}^{*}=k_{11} T, & m^{*}=m T .
\end{array}
$$

Inserting the dimensionless variables and parameters above into the system of equations (3.1) and after dropping the stars for notational convenience, the nondimensional model for cell-matrix adhesion is now

$$
\begin{aligned}
\frac{d x_{1}}{d t} & =\alpha k_{2} x_{3}-\frac{1}{\beta} k_{1} x_{1} x_{2} \\
\frac{d x_{2}}{d t} & =\beta k_{2} x_{3}-\frac{1}{\alpha} k_{1} x_{1} x_{2}-k_{7} x_{2} x_{4}, \\
\frac{d x_{3}}{d t} & =\frac{1}{\alpha \beta} k_{1} x_{1} x_{2}-k_{2} x_{3}-k_{3} x_{3}
\end{aligned}
$$




$$
\begin{aligned}
\frac{d x_{4}}{d t} & =k_{3} x_{3}+k_{6} x_{6}-\frac{1}{\alpha} k_{5} x_{4} x_{5}-\frac{1}{\beta} k_{7} x_{2} x_{4}+k_{11} x_{8}, \\
\frac{d x_{5}}{d t} & =k_{41} x_{9}-k_{42} x_{5}+\alpha k_{6} x_{6}-k_{5} x_{4} x_{5}-m x_{5}, \\
\frac{d x_{6}}{d t} & =\frac{1}{\alpha} k_{5} x_{4} x_{5}+k_{8} x_{7}-k_{6} x_{6}-k_{9} x_{6} x_{7}+k_{10} x_{8}, \\
\frac{d x_{7}}{d t} & =\frac{1}{\beta} k_{7} x_{2} x_{4}-k_{8} x_{7}+k_{10} x_{8}-k_{9} x_{6} x_{7}, \\
\frac{d x_{8}}{d t} & =k_{9} x_{6} x_{7}-k_{10} x_{8}-k_{11} x_{8} \\
\frac{d x_{9}}{d t} & =k_{42} x_{5}-k_{41} x_{9},
\end{aligned}
$$

where

$$
\alpha=\frac{k_{1}}{k_{2}} F_{T} \quad \text { and } \quad \beta=\frac{k_{1}}{k_{2}} R_{T} .
$$

For migrating cells, adhesion of cells to matrix occurs instantaneously in order to prevent the formation of mature focal adhesions which are bigger in size and may hold cells from moving.

\section{Computational Simulation Results}

In this section we present the results of computational simulations from the system (3.2). To obtain dimensionless parameter values, we use parameters listed in Table 1 for reference. The parameter values are given in ranges reflecting the range of different values obtained experimentally in different conditions and using different materials.

Table 1: Estimated parameter values

\begin{tabular}{cccc}
\hline Parameter & Definition & Range/Value & Reference \\
\hline$x_{R}$ & Receptor density & $10^{8}-10^{12} \mathrm{~cm}^{-2}$ & {$[35]$} \\
$x_{F}$ & Ligand density & $10^{6}-10^{13} \mathrm{~cm}^{-2}$ & {$[35]$} \\
$\frac{k_{1}}{k_{2}}$ & Receptor-ligand equilibrium constant & $10^{-8}-10^{-6} \mathrm{M}^{-1}$ & {$[35]$} \\
$k_{2}, k_{6}, k_{10}$ & Reverse reaction rates & $10^{-5}-10^{1} \mathrm{~s}^{-1}$ & {$[35]$} \\
$\frac{k_{5}}{k_{6}}$ & Receptor-cytoskeleton equilibrium constant & $10^{-8}-10^{-6} \mathrm{M}^{-1}$ & {$[35]$} \\
$T$ & Time integrin clusterings start at focal contacts & 1 hour & {$[23]$} \\
\hline
\end{tabular}

For parameter values that could not be found in the literature, we use estimated values. Using estimates in developing models of complex biochemical pathway is generally acknowledged to 
generate uncertainty. However, these values serve as tools for a preliminary study of the cellmatrix adhesion pathway and they are useful for simulation purposes. Taking

$$
\begin{array}{lll}
R_{T}=0.5 \times 10^{8} \text { molecules } / \mathrm{cm}^{2}, & k_{2}=0.5 \times 10^{-3} \mathrm{~s}^{-1}, & \frac{k_{1}}{k_{2}}=10^{-8} \mathrm{M}^{-1}, \quad T=3600 \mathrm{~s} \\
F_{T}=10^{8} \text { molecules } / \mathrm{cm}^{2}, & \frac{k_{5}}{k_{6}}=4 \times 10^{-8} \mathrm{M}^{-1}, & k_{6}=0.5 \times 10^{-4} \mathrm{~s}^{-1},
\end{array}
$$

we get dimensionless parameter values for (after omitting the star sign):

$$
\begin{array}{lll}
\alpha=1.0, & \beta=0.5, & k_{1}=0.9, \\
k_{2}=1.8, & k_{5}=0.36, & k_{6}=0.18
\end{array}
$$

while the rest of the parameters are estimated as

$$
\begin{array}{lll}
k_{3}=10, & k_{41}=2.15, & k_{42}=1.15, \\
k_{7}=3.2, & k_{8}=0.001, & k_{9}=5, \\
k_{10}=0.01, & k_{11}=0.004, & m=0.005 .
\end{array}
$$

Initially we assume that there is a 0.6 fraction of free integrins on the cell membrane, a 0.6 fraction of soluble fibronectin and a small amount ( 0.03 fraction) of fibronectin matrix outside the surface of the cell. As a cell must have a cytoskeleton to support its shape, we assume there is a 0.3 fraction of actin filaments near the leading edge and a 0.03 fraction of actin monomers. The rest of the variables are initially zero.
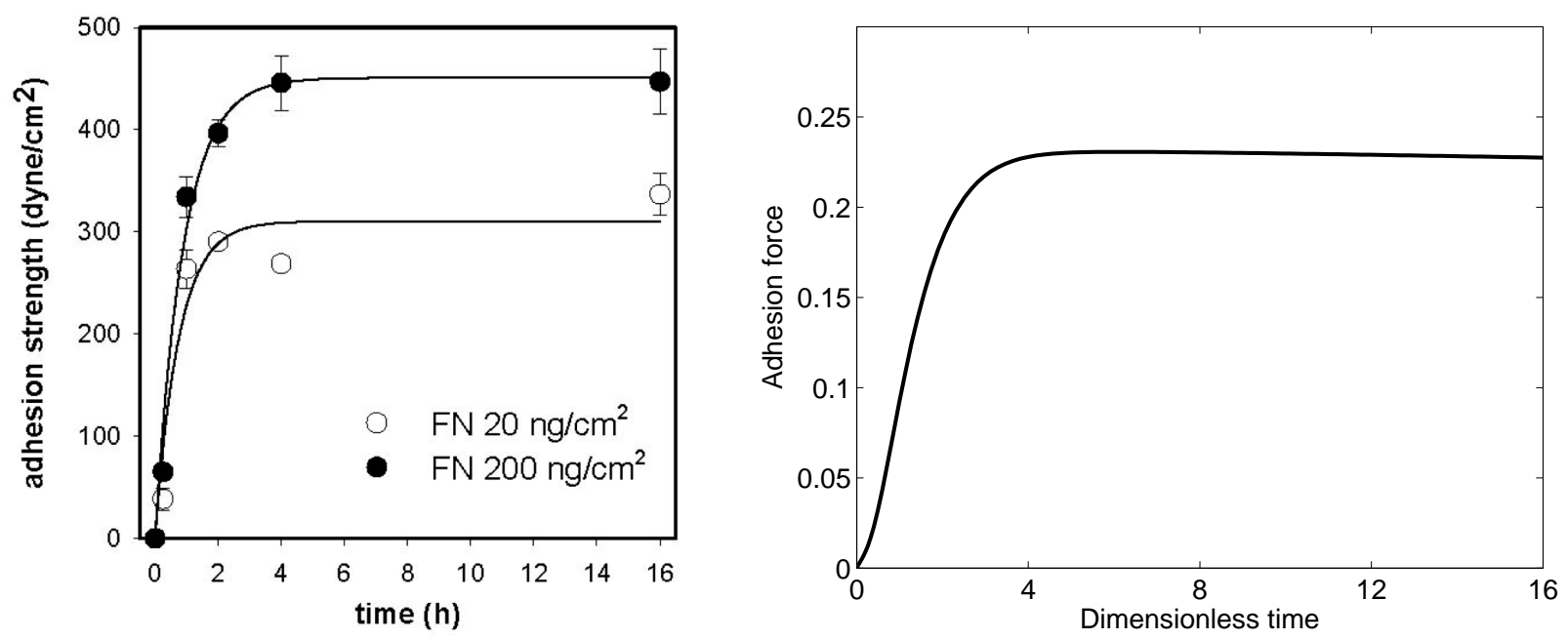

Figure 2: Plots showing a comparison between experimental data showing increase and steady state of adhesion force by [15] and our simulation result of generated adhesion force $x_{8}$ (right figure) from cell-matrix adhesion model (3.2) equation (3.2h). 
The first result from the cell-matrix adhesion pathway that we show here is the adhesion force or adhesion strength produced from complex interactions between the cell surface receptor integrins as mediators, the cytoskeletal network inside of the cells and fibronectin with the fibrillar network outside of the cell. Using the dimensionless parameters given above, the computational simulations show that the generated adhesion strength shows an initial exponential increase, until it reaches a steady state or a constant strength, as shown on the right plot of Fig. 2. This result qualitatively agrees with the experimental data by [15] that measured fibroblast adhesion strengthening on fibronectin, as in the left plot of Fig. 2.

Both experimental data and our simulation result show that the adhesion force or strength increases rapidly at early times and reaches a steady state value by 4 hours. Experiments by [15] show the dependency of adhesion strength on fibronectin density, where a lower density of fibronectin yields a smaller constant adhesion strength (open circles) and a higher fibronectin density gives a bigger constant adhesion strength (closed circles). For our cell-matrix adhesion model, the adhesion strength shows dependency both on integrin and soluble fibronectin densities. In Fig. 3 we plot adhesion strengths over time by varying the initial value of integrin $x_{1}(0)$ and soluble fibronectin $x_{2}(0)$ concentrations as shown on the left figure (for varying/increasing $x_{1}(0)$ ) and right figure (for varying/increasing $x_{2}(0)$ ). The initial value of concentrations is increased from $x_{1}(0)=0.1$ (bottom curves), $x_{1}(0)=0.3, x_{1}(0)=0.6$, and $x_{1}(0)=0.9$ (top curves) for both $x_{1}(0)$ and $x_{2}(0)$. Taking both $x_{1}(0)=0.9$ and $x_{2}(0)=0.9$, the time taken for the adhesion strength to reach a constant value is slightly different for both concentrations, also at around 4 dimensionless time units. The constant adhesion strengths are higher for initial densities of integrin than the initial densities of fibronection. With the lowest initial densities $x_{1}(0)=0.1$ and $x_{2}(0)=0.1$, the time taken for the adhesion strength to reach a constant value is longer, around 8 dimensionless time units and the constant values of adhesion strengths are different for each integrin and fibronectin, where with $x_{1}(0)=0.1$ the constant adhesion strength is around 0.17 and with $x_{2}(0)=0.1$ the constant adhesion strength is around 0.11 .

[6] developed an in vitro assay to visualise and quantify actin polymerisation from purified $\alpha_{v} \beta_{3}$ integrin complexes that cluster at the protruding parts of the cells as shown on the left plot of Fig. 4. The experimental data show the rates of actin assembly/polymerisation from different cells stimulated and unstimulated with Arg-Gly-Asp-ligand-induced $\beta_{3}$ tyrosine phosphorylation, where the black curve shows the rate of actin polymerisation by cells of wild type stimulated with Arg-Gly-Asp-ligand-induced $\beta_{3}$ tyrosine phosphorylation, the blue curve from unstimulated cells, the red curve for stimulated and expressing Y747-759F $\beta_{3}$ cells, and the orange curves for unstimulated expressing Y747-759F $\beta_{3}$ cells. The rate of actin polymerisation from experimental data shows a tendency of a rapid increase at early times until it reaches a constant value, the same pattern as the experimental data for adhesion strength. Our computational simulation result for $x_{5}$ from the model (3.2) which accounts for actin polymerisation followed by actin reorganisation near the leading edge as well as actin depolymerisation and retrograde flow for balance, also shows the same exponential increase until reaching a constant rate, as shown by the right plot of Fig. 4.

Other processes that are of experimental interest are the rate of change of soluble fibronectin and the rate of change of formation of insoluble cellular fibronectin or fibrillar fibronectin network (matrix) outside of the cell. We compare our simulation results for both kinetics with the 

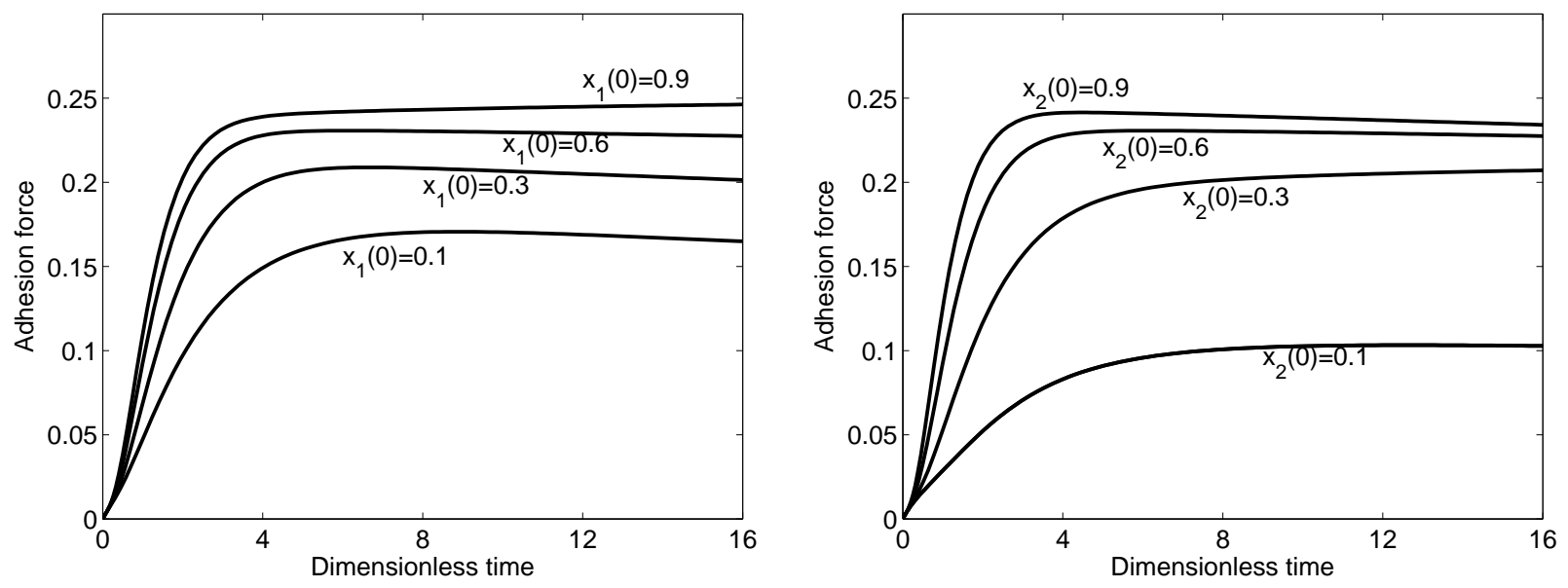

Figure 3: Plots showing increasing constant adhesion strength values by increasing initial concentration of integrins $x_{1}(0)$ (left figure) and initial concentration of soluble fibronectin $x_{2}(0)$ (right figure) from $0.1,0.3,0.6$, and 0.9 .
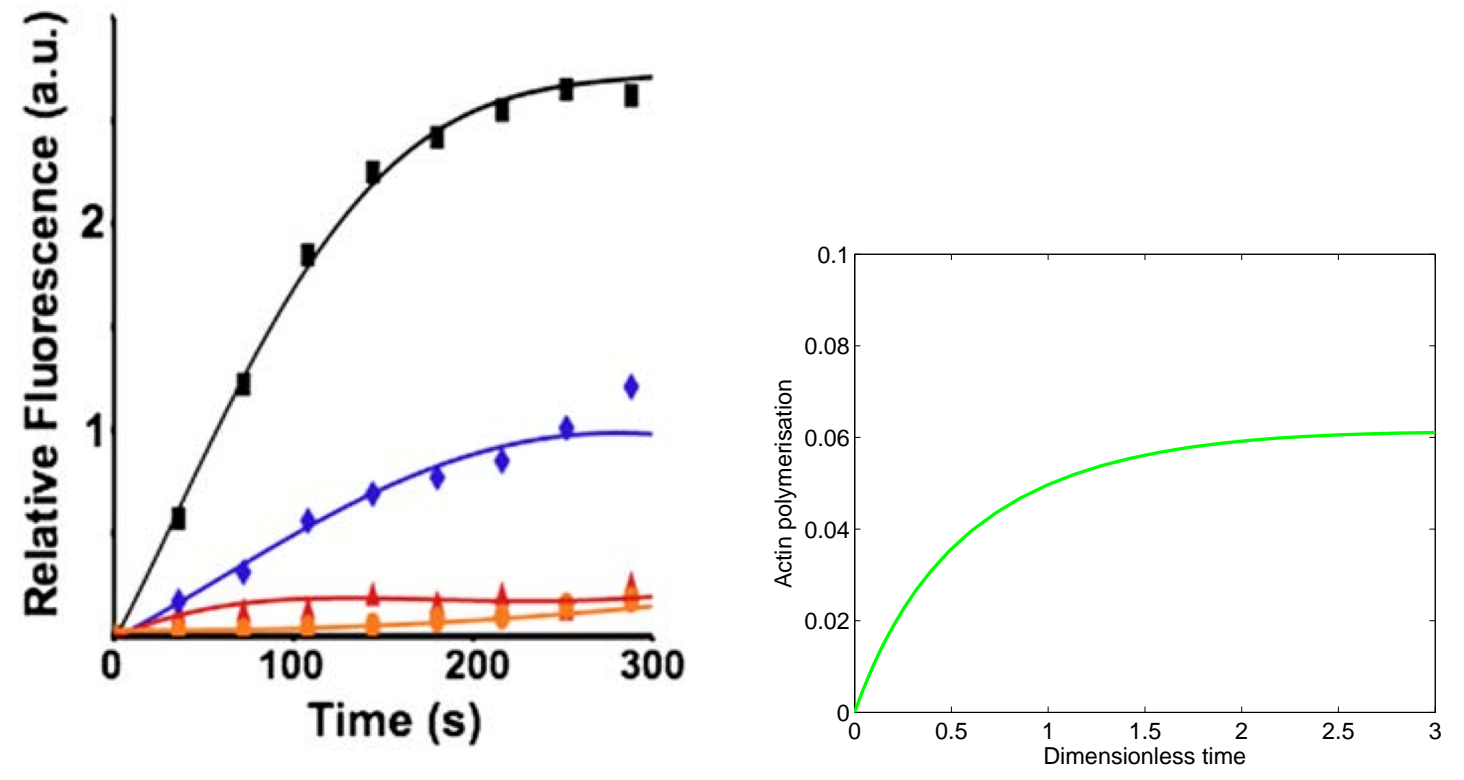

Figure 4: Plots showing different rates of actin polymerisation from experimental data by [6] (left figure) and simulation result of actin polymerisation $x_{5}$ from cell-matrix adhesion model of equation (3.2e) (right figure). Left figure is reprinted from Current Biology, 16/3, B. Butler, C. Gao, A.T. Mersich, S.D. Blystone, Purified integrin adhesion complexes exhibit actin-polymerization activity, 242-251, Copyright (2006), with permission from Elsevier [OR APPLICABLE SOCIETY COPYRIGHT OWNER]. 
experimental data of [44], as shown in Fig. 5. The left plot, which is the experimental data, shows the fast formation of insoluble fibronectin (open circles) from a certain type of soluble fibronectin (closed circles) (FN $\left.\Delta I I I_{1-7}\right)$ that rapidly decreases. The concentration of soluble and insoluble fibronectin decays and grows, respectively. The simulation results, shown by the right plot, from equations (3.2b) for soluble fibronectin concentration and $(3.2 \mathrm{~g})$ for insoluble fibronectin concentration exhibit the same rate of reactions, which are an exponential decrease for soluble fibronectin and an exponential increase for insoluble fibronectin.

\section{FN $\Delta \mathrm{IIII}_{1-7}$}
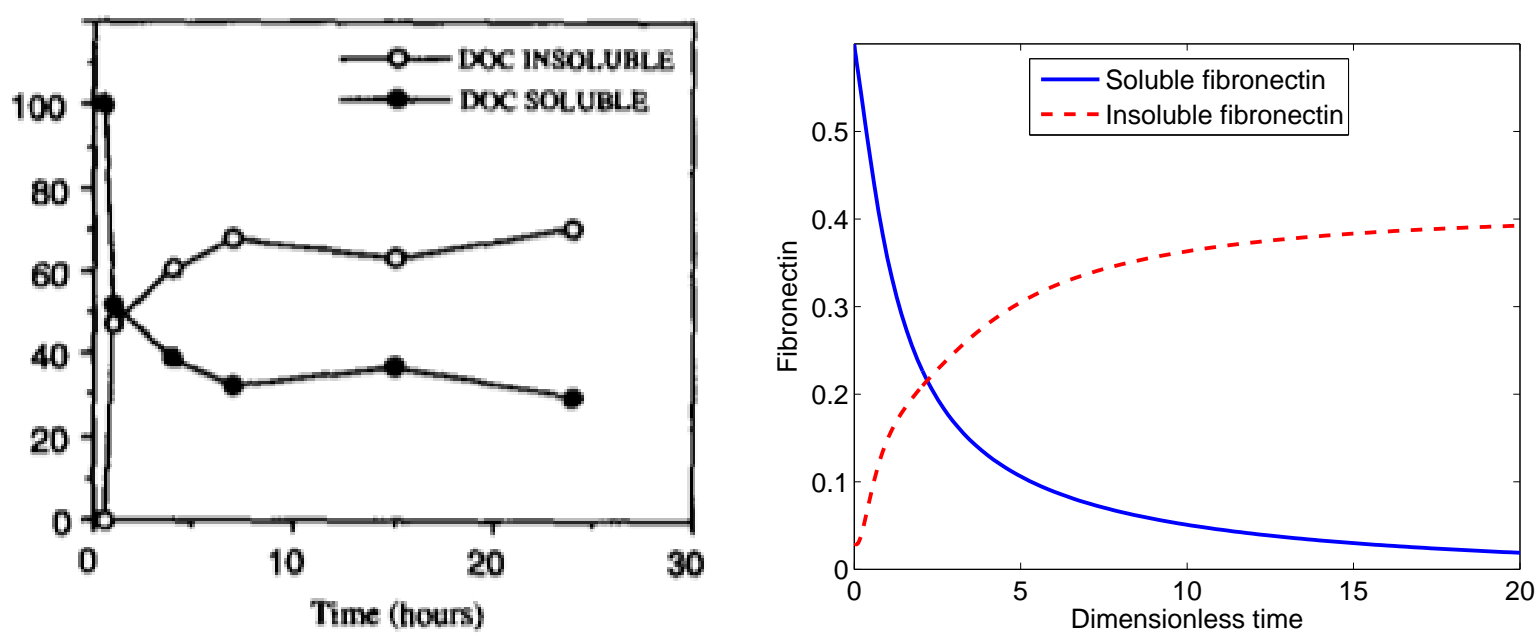

Figure 5: Plots showing the computational results of our model for kinetics of soluble fibronectin $x_{2}$ and the formation of insoluble fibronectin matrix $x_{7}$ (right figure) are qualitatively comparable with experimental data by [44] (left figure).

Some biochemical processes that are considered to be important, indeed crucial, to adhesion for cell motility are the integrin/fibronectin complex clustering and the binding between clustering and soluble fibronectin which is necessary for matrix formation. From our model of the system (3.2), if we prevent clustering of the integrin/fibronectin complex by setting $k_{3}=0$, the magnitude of the force decreases and it takes a longer time for the adhesion force to be stable (i.e. to reach a constant force). If we set $k_{7}=0$, the adhesion force becomes very low/weak, perhaps not enough to perform adhesion. The changes in the magnitude of adhesion force over time for both scenarios can be seen in figure Fig. 6.

Setting the parameters $k_{3}=0$ and $k_{7}=0$ as above also reduces the formation of fibrillar matrix, as can be seen in the left and right figures of Fig. 7, respectively. Even with $k_{7}=0$, no fibrillar matrix will be assembled/formed where the solution goes to zero.

To explore in more detail how changing the key parameters above affect the dynamics, we performed a parameter sensitivity analysis using the Systems Biology Toolbox or SBToolbox $\mathbb{R}$ [43] which is a Matlabßtoolbox. We determined the sensitivities of the integrin/fibronectin com- 


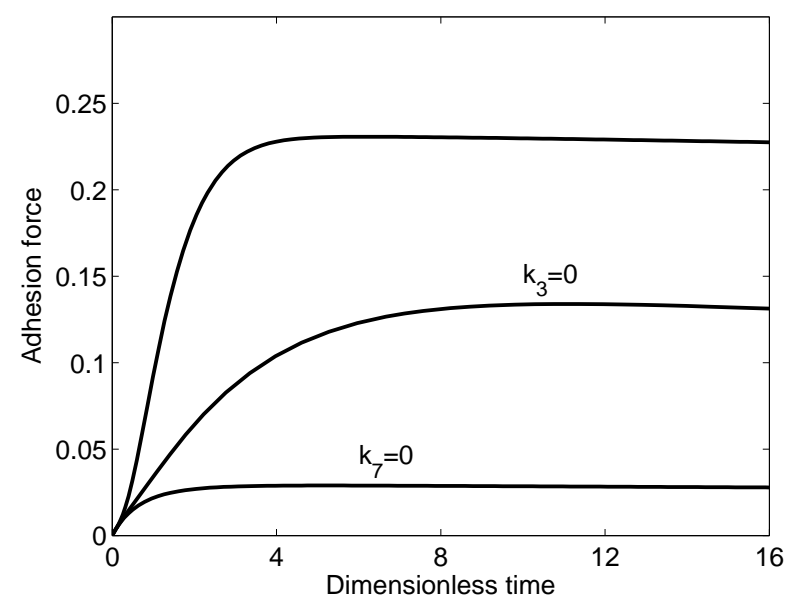

Figure 6: Plots showing decreases of the adhesion strength if (i) the clustering of integrin/fibronectin complex is prevented by setting $k_{3}=0$ (middle curve), and (ii) the formation of fibronectin matrix is deprived by setting $k_{7}=0$ (bottom curve) as compared to the top curve for default parameter values.
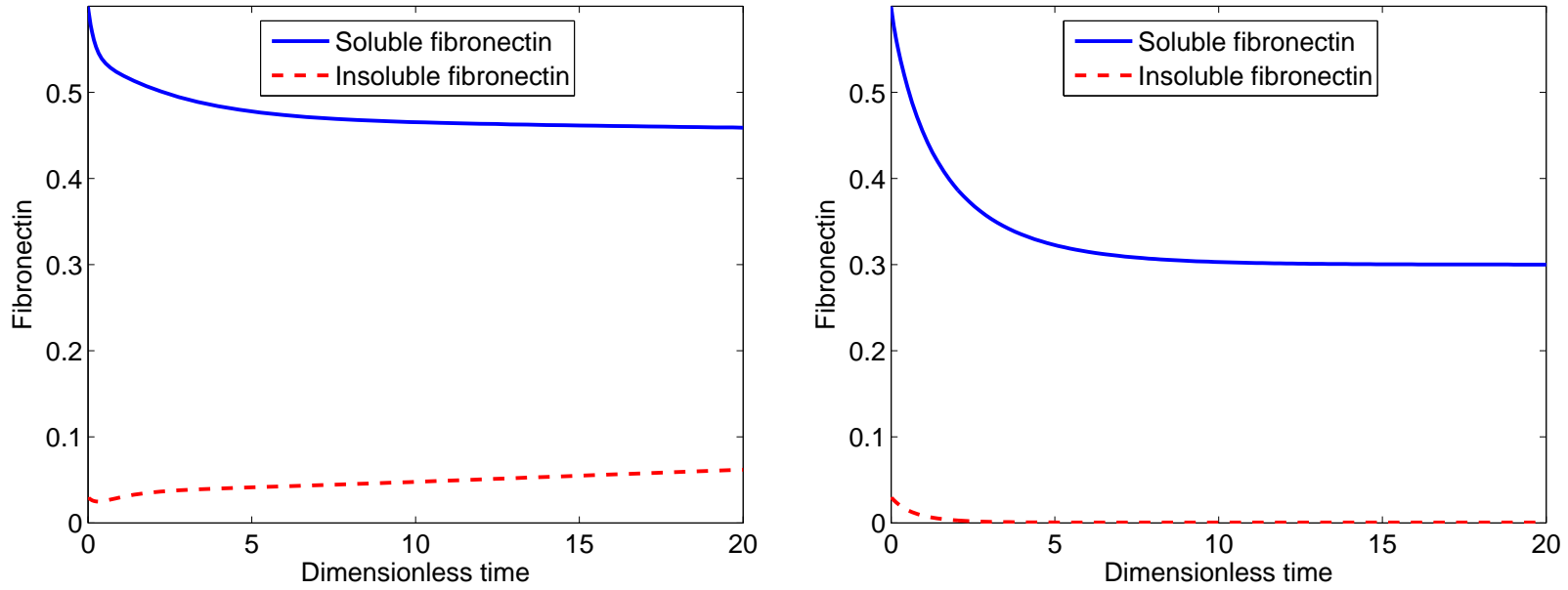

Figure 7: Plots showing the effect on the formation of insoluble fibronectin matrix by setting $k_{3}=0$ (left figure) and $k_{7}=0$ (right figure).

plex clustering (through the variable $k_{3} x_{3}$ ) and the fibronectin matrix formation (through the variable $k_{7} x_{2} x_{4}$ ) to the parameters. The sensitivity analysis was performed by perturbing all parameters to obtain the steady-state sensitivities. As shown in Fig. 8 variations in both key processes generate (almost) identical values of steady-state sensitivities of the parameters, and parameters $\beta, k_{8}$ and $\alpha$ are the most sensitive to the variations. 

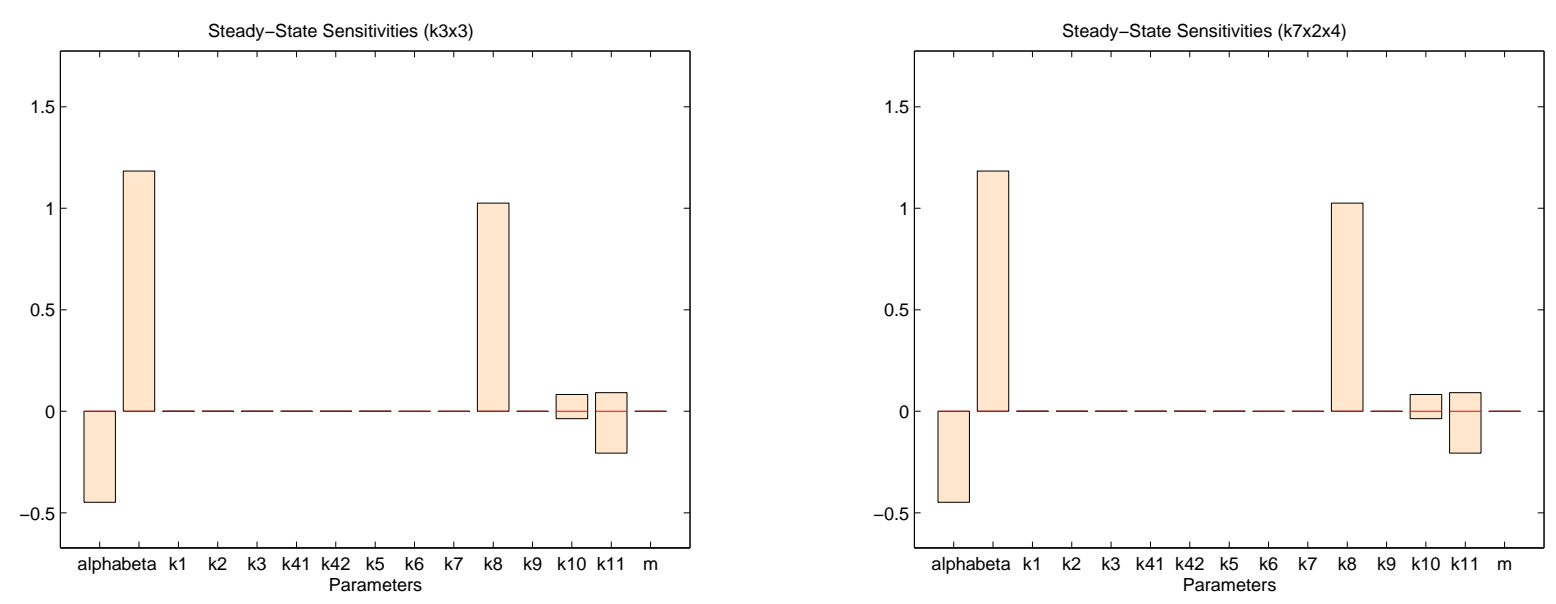

Figure 8: Plots showing steady-state sensitivities to the reactions $k_{3} x_{3}$ for integrin/fibronectin complex clustering (left figure) and $k_{7} x_{2} x_{4}$ for the formation of fibronectin matrix (right figure). As can be seen from the figures, parameters $\beta, k_{8}$ and $\alpha$ are the most sensitive to the variations.

\section{Conclusions and Discussion}

In this paper we have presented a model of the key biochemical processes involved in cell-matrix adhesion that accounts for reactions between integrins, soluble fibronectin, the integrin/fibronectin complex, clustering of the integrin/fibronectin complex, actin polymerisation and reorganisation, the cytoskeletal complex, insoluble fibronectin (fibrillar network), and adhesion strength from the combination of the clustering integrin/fibronectin complex with the cytoskeletal network and attachment to insoluble fibronectin outside the cell, using a system of ordinary differential equations (3.2). We formulated the mathematical model from a proposed cell-matrix adhesion pathway that was derived based on the key events as reported in the literature.

Despite some parameters used in the simulations are only estimated values because not all parameters are available experimentally or in literature, our model shows good qualitative agreement with the experimental data, such as for adhesion strength plots shown in Figs. 2 and 3 and for kinetics of soluble fibronectin and formation of matrix fibronectin as shown in Fig. 5.

For migrating cells, the adhesion force generated from interactions of proteins locally is used for one cycle of movement which includes extending protrusions and moving their body forward. One cycle of cell movement takes around 30 minutes to 1 hour to occur, depending on many other variables such as a sufficient rigidity of surface/matrix for the smooth movement, an available gradient of chemoattractant, etc. The dynamics of key cell-adhesion components such as integrins, the cytoskeletal network and fibronectin, are also instantaneous. After one cycle of movement is completed, the whole pathway is restarted from the beginning to perform another cycle of movement. In order to prove this conjecture and to see whether our cell-matrix adhesion pathway works adequately, it is our aim for future work to apply the intracellular cell-matrix adhesion model into 
a multiscale cellular model.

\section{Acknowledgements}

MAJC gratefully acknowledges the support of ERC (European Research Council) AdG Grant No. 227619 "From Mutations to Metastases: Multiscale Mathematical Modelling of Cancer Growth and Spread".

\section{References}

[1] V.C. Abraham, V. Krishnamurthi, D.L. Taylor, F. Lanni. The actin-based nanomachine at the leading edge of migrating cells. Biophys. J., 77 (1999), No. 3, 1721-1732.

[2] O. Ali, H. Guillou, O. Destaing, C. Albiges-Rizo, M.R. Block, B. Fourcade. Cooperativity between integrin activation and mechanical stress leads to integrin clustering. Biophys. J., 100 (2011), No. 11, 2595-2604.

[3] M. Amano, K. Chihara, K. Kimura, Y. Fukata, N. Nakamura, Y. Matsuura, K. Kaibuchi. Formation of actin stress fibers and focal adhesions enhanced by rho-kinase. Science, 297 (1997), No. 5304, 1308-1311.

[4] R. Ananthakrishnan, A. Ehrlicher. The force behind cell movement. Int. J. Biol. Sci., 3 (2007), No. 5, 303-317.

[5] A.L. Berrier, K.M. Yamada. Cell-matrix adhesion. J. Cell. Physiol., 213 (2007), No. 3, 565573.

[6] B. Butler, C. Gao, A.T. Mersich, S.D. Blystone. Purified integrin adhesion complexes exhibit actin-polymerization activity. Curr. Biol., 16 (2006), No. 3, 242-251.

[7] L.L. Chen, A. Whitty, R.R. Lobb, S.P. Adams, R.B. Pepinsky. Multiple activation states of integrin $\alpha_{4} \beta_{1}$ detected through their different affinities for a small molecule ligand. J. Biol. Chem., 274 (1999), No. 19, 13167-13175.

[8] D. Choquet, D.P. Felsenfeld, M.P. Sheetz. Extracellular matrix rigidity causes strengthening of integrin-cytoskeleton linkages. Cell, 88 (1997), No. 1, 39-48.

[9] C. Cluzel, F. Saltel, J. Lussi, F. Paulhe, B.A. Imhof, B. Wehrle-Haller. The mechanisms and dynamics of $\alpha_{v} \beta_{3}$ integrin clustering in living cells. J. Cell. Biol., 171 (2005), No. 2, 383-392.

[10] B. Cseh, S. Fernandez-Sauze, D. Grall, S. Schaub, E. Doma, E. van Obberghen-Schilling. Autocrine fibronectin directs matrix assembly and crosstalk between cell-matrix and cell-cell adhesion in vascular endothelial cells. J. Cell Sci., 123 (2010), No. 22, 3989-3999. 
[11] P.A. DiMilla, K. Barbee, D.A. Lauffenburger. Mathematical model for the effects of adhesion and mechanics on cell migration speed. Biophys. J., 60 (1991), No. 1, 15-37.

[12] G.J. Doherty, M.K. Ahlund, M.T. Howes, B. Moren, R.G. Parton, H.T. McMahon, R. Lundmark. The endocytic protein GRAF1 is directed to cell-matrix adhesion sites and regulates cell spreading. Mol. Biol. Cell, 22 (2011), No. 22, 4380-4389.

[13] P. Friedl, K. Wolf. Tumour-cell invasion and migration: diversity and escape mechanisms. Nat. Rev. Cancer, 3 (2003), No. 5, 362-374.

[14] M. Fussenegger, J.E. Bailey, J. Varner. A mathematical model of caspase function in apoptosis. Nat. Biotechnol., 18 (2000), 768-774.

[15] N.D. Gallant, K.E. Michael, A.J. García. Cell adhesion strengthening: contributions of adhesive area, integrin binding, and focal adhesion assembly. Mol. Biol. Cell, 16 (2005), No. 9, 4329-4340.

[16] A.J. García, D. Boettiger. Integrin-fibronectin interactions at the cell-material interface: initial integrin binding and signaling. Biomaterials, 20 (1999), No. 23-24, 2427-2433.

[17] A.J. García, F. Huber, D. Boettiger. Force required to break $\alpha_{5} \beta_{1}$ integrin-fibronectin bonds in intact adherent cells is sensitive to integrin activation state. J. Biol. Chem., 273 (1998), No. 18, 10988-10993.

[18] F.G. Giancotti, E. Ruoslahti. Integrin signaling. Science, 285 (1999), No. 5430, 1028-1032.

[19] M.Z. Gilcrease, X. Zhou, K. Welch. Adhesion-independent $\alpha_{6} \beta_{4}$ integrin clustering is mediated by phosphatidylinositol 3-kinase. Cancer Res., 64 (2004), 7395.

[20] W.H. Guo, Y.L. Wang. Retrograde fluxes of focal adhesion proteins in response to cell migration and mechanical signals. Mol. Biol. Cell, 18 (2007), No. 11, 4519-4527.

[21] D.A. Hammer, D.A. Lauffenburger. A dynamical model for receptor-mediated cell adhesion to surfaces. Biophys. J., 53 (1987), No. 3, 475-487.

[22] R.O. Hynes. Integrins: bidirectional, allosteric signaling machines. Cell, 110 (2002), No. 6, 673-687.

[23] K. Kawakami, H. Tatsumi, M. Sokabe. Dynamics of integrin clustering at focal contacts of endothelial cells studied by multimode imaging microscopy. J. Cell Sci., 114 (2001), No. 17, 3125-3135.

[24] P. Koistinen, J. Heino. Integrins in cancer cell invasion. Cell invasion. Landes Bioscience, 2002.

[25] Z.H. Li, M. Kreiner, C.F. van der Walle, H.J. Mardon. Clustered integrin alpha-5-beta-1 ligand displays model fibronectin-mediated adhesion of human endometrial stromal cells. Biochem. Biophys. Res. Comm., 407 (2011), No. 4, 777-782. 
[26] L.M. Machesky, A. Hall. Role of actin polymerization and adhesion to extracellular matrix in Rac-and Rho-induced cytoskeletal reorganization. J. Cell. Biol., 138 (1997), No. 4, 913-926.

[27] A. Mallavarapu, T. Mitchison. Regulated actin cytoskeleton assembly at filopodium tips controls their extension and retraction. J. Cell. Biol., 146 (1999), No. 5, 1097-1106.

[28] M. Martini, A. Gnann, D. Scheiki, B. Holzmann, K.P. Janssen. The candidate tumor suppressor SASH1 interacts with the actin cytoskeleton and stimulates cell-matrix adhesion. Int. J. Biochem. Cell. Biol., 43 (2011), No. 11, 1630-1640.

[29] E. Monaghan, V. Gueorguiev, C. Wilkins-Port. The receptor for urokinase-type plasminogen activator regulates fibronectin matrix assembly in human skin fibroblasts. J. Biol. Chem., 279 (2004), No. 2, 1400-1407.

[30] F.A. Moretti, A.K. Chauhan, A. Iaconcig, F. Porro, F.E. Baralle, A.F. Muro. A major fraction of fibronectin present in the extracellular matrix of tissues is plasma-derived. J. Biol. Chem., 282 (2007), No. 38, 28057-28062.

[31] S. Niland, J.A. Eble. Integrin-mediated cell-matrix interaction in physiological and pathological blood vessel formation. J. Oncol., (2012), Epub 2011 Sep 18, 125278.

[32] T. Nishizaka, Q. Shi, M.P. Sheetz. Position-dependent linkages of fibronectin-integrincytoskeleton. PNAS, 97 (2000), No. 2, 692-697.

[33] M. Ojaniemi, K. Vuori. Epidermal growth factor modulates tyrosine phosphorylation of p130Cas. Involvement of phophatidylinositol 3'-kinase and actin cytoskeleton. J. Biol. Chem., 272 (1997), No. 41, 25993-25998.

[34] T. Osada, Y.H. Gu, M. Kanazawa, Y. Tsubota, B.T. Hawkins, M. Spatz, R. Milner, G.J. del Zoppo. Interendothelial claudin-5 expression depends on cerebral endothelial cell-matrix adhesion by beta(1)-integrins. J. Cereb. Blood Flow Metab., 31 (2011), No. 10, 1972-1985.

[35] S.P. Palecek, A.F. Horwitz, D.A. Lauffenburger. Kinetic model for integrin-mediated adhesion release during cell migration. Ann. Biomed. Eng., 27 (1999), No. 2, 219-235.

[36] S.P. Palecek, A. Huttenlocher, A.F. Horwitz, D.A. Lauffenburger. Physical and biochemical regulation of integrin release during rear detachment of migrating cells. J. Cell Sci., 111 (1998), 929-940.

[37] S.P. Palecek, J.C. Loftus, M.H. Ginsberg, D.A. Lauffenburger, A.F. Horwitz. Integrin-ligand binding properties govern cell migration speed through cell-substratum adhesiveness. Nature, 385 (1997), No. 6616, 537-540.

[38] R. Pankov, K.M. Yamada. Fibronectin at a glance. J. Cell Sci., 115 (2002), No. 20, 38613863. 
[39] M.J. Paszek, D. Boettiger, V.M. Weaver, D.A. Hammer. Integrin clustering is driven by mechanical resistance from the glycocalyx and the substrate. PLoS Comput. Biol., 5 (2009), No. 12, e1000604.

[40] T.D. Pollard, M.S. Mooseker. Direct measurement of actin polymerization rate constants by electron microscopy of actin filaments nucleated by isolated microvillus cores. J. Cell Biol., 88 (1981), No. 3, 654-659.

[41] C.M. Regen, A.F. Horwitz. Dynamics of $\beta_{1}$ integrin-mediated adhesive contacts in motile fibroblasts. J. Cell Biol., 119 (1992), No. 5, 1347-1359.

[42] S. Roy, L. Bingle, J.F. Marshall, R. Bass, V. Ellis, P.M. Speight, S.A. Whawell. The role of alpha 9 beta 1 integrin in modulating epithelial cell behaviour. J. Oral Pathol., 40 (2011), No. 10, 755-761.

[43] H. Schmidt, M. Jirstrand. Systems Biology Toolbox for MATLAB: a computational platform for research in systems biology. Bioinformatics, 22 (2006), No. 4, 514-515.

[44] J.L. Sechler, Y. Takada, J.E. Schwarzbauer. Altered rate of fibronectin matrix assembly by deletion of the first type III repeats. J. Cell Biol., 134 (1996), No. 2, 573-583.

[45] D.S. Spassov, C.H. Wong, N. Sergina, D. Ahuja, M. Fried, D. Sheppard, M.M. Moasser. Phosphorylation of trask by src kinases inhibits integrin clustering and functions in exclusion with focal adhesion signaling. Mol. Cell. Biol., 31 (2011), No. 4, 766-782.

[46] Y. Takada, X. Ye, S. Simon. The integrins. Genome Biol., 8 (2007), No. 5, 215.

[47] J.W. Tamkun, R.O. Hynes. Plasma fibronectin is synthesized and secreted by hepatocytes. J. Biol. Chem., 258 (1983), No. 7, 4641-4647.

[48] M. Waldeck-Weiermair, C. Zoratti, K. Osibow, N. Balenga, E. Goessnitzer, M. Waldhoer, R. Malli, W.F. Graier. Integrin clustering enables anandamide-induced Ca2+ signaling in endothelial cells via GPR55 by protection against CB1-receptor-triggered repression. J. Cell Sci., 121 (2008), 1704-1717.

[49] D.J. Webb, J.T. Parsons, A.R. Horwitz. Adhesion assembly, disassembly and turnover in migrating cells - over and over and over again. Nat. Cell Biol., 4 (2002), No. 4, E97-E100.

[50] B. Wehrle-Haller. Analysis of integrin dynamics by fluorescence recovery after photobleaching. Adhesion Protein Protocols. Springer, 2007.

[51] B. Wehrle-Haller, B.A. Imhof. Actin, microtubules and focal adhesion dynamics during cell migration. Int. J. Biochem. Cell Biol., 35 (2003), No. 1, 39-50.

[52] E.S. Welf, B.A. Ogunnaike, U.P. Naik. Quantitative statistical description of integrin clusters in adherent cells. IET Sys. Biol., 3 (2009), No. 5, 307-316. 
[53] I. Wierzbicka-Patynowski, J. Schwarzbauer. The ins and outs of fibronectin matrix assembly. J. Cell Sci., 116 (2003), No. 16, 3269-3276.

[54] P.W. Wiseman, C.M. Brown, D.J. Webb, B. Hebert, N.L. Johnson, J.A. Squier, M.H. Ellisman, A.F. Horwitz. Spatial mapping of integrin interactions and dynamics during cell migration by image correlation microscopy. J. Cell Sci., 117 (2004), No. 23, 5521-5534.

[55] T. Yu, X. Wu, K.B. Gupta, D.F. Kucik. Affinity, lateral mobility, and clustering contribute independently to $\beta_{2}$-integrin-mediated adhesion. Am. J. Physiol. Cell Physiol., 299 (2010), No. 2, C399-C410.

[56] F. Zhang, J.E. Michaelson, S. Moshiach, N. Sachs, W. Zhao, Y. Sun, A. Sonnenberg, J.M. Lahti, H. Huang, X.A. Zhang. Tetraspanin CD151 maintains vascular stability by balancing the forces of cell adhesion and cytoskeletal tension. Blood, 118 (2011), No. 15, 42744284. 